\title{
Constraining modified gravitational theories by weak lensing with Euclid
}

\author{
Matteo Martinelli, ${ }^{1}$ Erminia Calabrese,${ }^{1}$ Francesco De Bernardis, ${ }^{1}$ Alessandro Melchiorri, ${ }^{1}$ \\ Luca Pagano, ${ }^{1}$ and Roberto Scaramella ${ }^{2}$ \\ ${ }^{1}$ Physics Department and INFN, Universita' di Roma “La Sapienza”, Ple Aldo Moro 2, 00185, Rome, Italy \\ ${ }^{2}$ INAF, Osservatorio Astronomico di Roma, via Frascati 33, 0040 Monte Porzio Catone (RM), Italy \\ (Received 26 October 2010; revised manuscript received 21 December 2010; published 19 January 2011)
}

\begin{abstract}
Future proposed satellite missions such as Euclid can offer the opportunity to test general relativity on cosmic scales through mapping of the galaxy weak-lensing signal. In this paper we forecast the ability of these experiments to constrain modified gravity scenarios such as those predicted by scalar-tensor and $f(R)$ theories. We find that Euclid will improve constraints expected from the Planck satellite on these modified theories of gravity by 2 orders of magnitude. We discuss parameter degeneracies and the possible biases introduced by modifications to gravity.
\end{abstract}

DOI: 10.1103/PhysRevD.83.023012

PACS numbers: 98.70.Vc, 95.35.+d

\section{INTRODUCTION}

Understanding the nature of the current observed accelerated expansion of our Universe is probably the major goal of modern cosmology. Two possible mechanisms can be at work: either our Universe is described by general relativity (GR, hereafter) and its energy content is dominated by a negative pressure component, coined "dark energy," or only "standard" forms of matter exist and the cosmic acceleration is driven by deviations from GR on cosmic scales (see e.g. [1,2]) or arises because of large scale inhomogeneities (see e.g. $[3,4]$ ).

All current cosmological data are consistent with the choice of a cosmological constant as a dark energy component with equation of state $w=P / \rho=-1$, where $P$ and $\rho$ are the dark energy pressure and density, respectively (see e.g. [5-7]).

While deviations at the level of $\sim 10 \%$ on $w$ assumed as constant are still compatible with observations and bounds on $w$ are even weaker if $w$ is assumed to be redshift dependent, it may well be that future measurements will be unable to significantly rule out the cosmological constant value of $w=-1$.

Measuring $w$, however, is just part of the story. While the background expansion of the Universe will be identical to the one expected in the case of a cosmological constant, the growth of structures with time could be significantly different if GR is violated. Modified theories of gravity have recently been proposed where the expansion of the Universe is identical to the one produced by a cosmological constant, but where the primordial perturbations that will result in the large scale structures in the Universe we observed today grow at a different rate (see e.g. [8-10], the review [11], and references therein).

Weak-lensing measurements offer a great opportunity to map the growth of perturbations since they relate directly to the dark matter distribution and are not plagued by galaxy luminous bias [12-14]. Recent works have indeed made use of current weak-lensing measurements, combined with other cosmological observables, to constrain modifications to gravity yielding no indications for deviations from GR [15-20].

The next proposed satellite missions such as Euclid [21,22] or the Wide-Field Infrared Survey Telescope [23] could measure the cosmological weak-lensing signal to high precision, providing a detailed history of structure formation and the possibility to test GR on cosmic scales.

In this paper we study the ability of these future satellite missions to constrain modified theories of gravity and to possibly falsify a cosmological constant scenario. With respect to recent papers that have analyzed this possibility (e.g. $[24,25])$ we improve on several aspects. First of all, we forecast the future constraints by making use of Monte Carlo simulations on synthetic realizations of data sets. Previous analyses (see e.g. $[9,26,27]$ ) often used the Fisher matrix formalism which, while fast, may lose its reliability when Gaussianity is not respected due, for instance, to strong parameter degeneracies. Second, we properly include the future constraints achievable by the Planck satellite experiment, also considering CMB lensing, that is a sensitive probe of gravity modifications (see e.g. [28,29] and references therein). Third, we discuss the parameter degeneracies and the impact of modified theories of gravity on the determination of cosmological parameters. Finally, we focus on $f(R)$ and scalar-tensor theories, using the general parametrization proposed by [26].

Our paper is structured as follows. In Sec. II we introduce the parametrization used to describe departures from GR, and then specialize to the case of $f(R)$ and scalartensor theories. In Sec. III we describe galaxy weak lensing, while in Sec. IV we discuss how to extract lensing information from $\mathrm{CMB}$ data. We review the analysis method and the data forecasting in Sec. V. In Sec. VI we present our results, and we derive our conclusions in Sec. VII. 


\section{PARAMETRIZED GRAVITY MODIFICATIONS}

In this section we describe the formalism we use to parametrize departures from general relativity.

\section{A. Background expansion}

In the following analysis we fix the background expansion to a standard $\Lambda$ CDM cosmological model. The $\Lambda \mathrm{CDM}$ scenario is currently the best fit to available SN-Ia luminosity distance data and popular modified theories of gravity, e.g. $f(R)$, closely mimic $\Lambda \mathrm{CDM}$ at the background level with differences which are typically smaller than the precision achievable with geometric tests [30]. The most significant departures happen at the level of growth of structure and, by restricting ourselves to $\Lambda \mathrm{CDM}$ backgrounds, we are able isolate them.

\section{B. Structure formation}

In modified theories of gravity we expect departures from the standard growth of structure, even when the expansion history matches exactly the $\Lambda \mathrm{CDM}$ one. Let us consider the perturbed Friedmann-Robertson-Walker metric in longitudinal gauge (neglecting vector and tensor perturbations):

$$
d s^{2}=(1+2 \Psi) d t^{2}-(1-2 \Phi) \delta_{i j} d x^{i} d x^{j},
$$

where $\Phi$ and $\Psi$ are the Newtonian and metric potentials. A modified theory of gravity changes the evolution of perturbations, dark matter clustering, as well as the evolution of the potentials which can be scale dependent. In order to follow the growth of perturbations in modified theories of gravity, we employ the MGCAMB code developed in [26] (and publicly available; see Ref. [31]). In this approach the modifications to the Poisson and anisotropy equations are parametrized by two functions $\mu(a, k)$ and $\gamma(a, k)$ defined by

$$
\begin{gathered}
k^{2} \Psi=-\frac{a^{2}}{2 M_{P}^{2}} \mu(a, k) \rho \Delta, \\
\Phi=\gamma(a, k) \Psi,
\end{gathered}
$$

where $\rho \Delta \equiv \rho \delta+3 \frac{a H}{k}(\rho+P) v$ is the comoving density perturbation. In the modified gravity scenario an effective anisotropic stress could indeed arise and the two potentials appearing in the metric element, $\Phi$ and $\Psi$, are not necessarily equal, as in the $\Lambda \mathrm{CDM}$ model when the relativistic energy component is neglected. These functions can be expressed using the parametrization introduced by [32] (and used in [26]):

$$
\mu(a, k)=\frac{1+\beta_{1} \lambda_{1}^{2} k^{2} a^{s}}{1+\lambda_{1}^{2} k^{2} a^{s}},
$$

$$
\gamma(a, k)=\frac{1+\beta_{2} \lambda_{2}^{2} k^{2} a^{s}}{1+\lambda_{2}^{2} k^{2} a^{s}}
$$

where the parameters $\beta_{i}$ can be thought of as dimensionless couplings, $\lambda_{i}$ as dimensionful length scales, and $s$ is determined by the time evolution of the characteristic length scale of the theory. $\Lambda \mathrm{CDM}$ cosmology is recovered for $\beta_{1,2}=1$ or $\lambda_{1,2}^{2}=0 \mathrm{Mpc}^{2}$.

\section{Scalar-tensor theories}

This parametrization can be used to constrain chameleon-type scalar-tensor theories, where the gravity Lagrangian is modified with the introduction of a scalar field [33]. As shown in [26], for these kinds of theories the parameters $\left\{\beta_{i}, \lambda_{i}^{2}\right\}$ are related in the following way:

$$
\beta_{1}=\frac{\lambda_{2}^{2}}{\lambda_{1}^{2}}=2-\beta_{2} \frac{\lambda_{2}^{2}}{\lambda_{1}^{2}}
$$

and $1 \lesssim s \lesssim 4$.

This implies that we can analyze scalar-tensor theories adding three independent parameters to the standard cosmological parameter set.

\section{2. $f(R)$ theories}

Scalar-tensor theories of gravity and $f(R)$ theories are dynamically equivalent (at both quantum and classical levels; see e.g. [34]); in fact, $f(R)$ models can be thought of as a specific class of scalar-tensor theories. Nevertheless, in this paper, in addition to scalar-tensor models, we specifically consider cosmologically viable $f(R)$ theories that reproduce the $\Lambda \mathrm{CDM}$ background expansion as, using the parametrization described above, they allow us to work with less additional parameters than general scalar-tensor theories. In fact, in the specific case of $f(R)$ theories we can indeed additionally reduce the number of free parameters since $f(R)$ theories correspond to a fixed coupling $\beta_{1}=4 / 3$ [35]. Moreover, to have a cosmologically viable theory, the $s$ parameter must be $\sim 4$ [26]. The parametrization in Eq. (4) effectively neglects a factor representing the rescaling of Newton's constant [e.g. $\left(1+f_{R}\right)^{-1}$ in $f(R)$ theories] that, as pointed out in [36], is very close to unity in models that satisfy local tests of gravity [30] and thus negligible. However, when studying the $f(R)$ case, we need to include it to get a more precise Monte Carlo Markov chain (MCMC) analysis [see [36] for the detailed expression of Eq. (4)]. Even with this extended parametrization, we have only one free parameter left, the length scale $\lambda_{1}$. In this work we will constrain $f(R)$ theories through this parameter, evaluating the effects of these theories on gravitational lensing.

\section{GALAXY WEAK LENSING}

Weak gravitational lensing of the images of distant galaxies offers a useful geometrical way to map the matter distribution in the Universe. Following [12] one can 
describe the distortion of the images of distant galaxies through the tensor

$$
\psi_{i j}=\left(\begin{array}{cc}
-\kappa-\gamma_{1} & -\gamma_{2} \\
-\gamma_{2} & -\kappa+\gamma_{2}
\end{array}\right),
$$

where $\kappa$ is the convergence term and $\gamma=\gamma_{1}+i \gamma_{2}$ is the complex shear field. As shown in [37] the shear and the convergence terms can be written as a function of the projected Newtonian potentials $\psi_{, i j}$ :

$$
\begin{gathered}
\gamma=\frac{1}{2}\left(\psi_{, 11}-\psi_{, 22}\right)+i \psi_{, 12}, \\
k=\frac{1}{2}\left(\psi_{, 11}-\psi_{, 22}\right),
\end{gathered}
$$

where the commas indicate the derivatives with respect to the directions transverse to the line of sight and the projected potentials are $\psi_{, i j}=-(1 / 2) \int g(z)\left(\Psi_{, i j}+\Phi_{, i j}\right) d z$ with the lensing kernel

$$
g(z)=\int d z^{\prime} \frac{n\left(z^{\prime}\right) D_{A}\left(z, z^{\prime}\right)}{D_{A}\left(0, z^{\prime}\right)} .
$$

Here $n(z)$ is the galaxy redshift distribution. In our analysis we assume flatness of the Universe. However, in general, the angular diameter distance $D_{A}$ between the lens and the source depends on the spatial curvature $K$ :

$$
\begin{gathered}
D_{A}=\frac{1}{\sqrt{K}} \sin (\sqrt{K} r), \quad K>0, \\
D_{A}=r, \quad K=0, \\
D_{A}=\frac{1}{\sqrt{-K}} \sinh (\sqrt{-K} r), \quad K<0,
\end{gathered}
$$

and the comoving distance is

$$
r\left(z, z^{\prime}\right)=\int_{z}^{z^{\prime}} \frac{d z^{\prime}}{E\left(z^{\prime}\right)}
$$

with $E(z)=H(z) / H_{0}$.

Image distortions induced by the matter distribution are generally small. To extract cosmological information it is hence necessary to statistically analyze a large number of images. The two-point correlation function of the convergence is, at present, the best measured statistic of the weak lensing but, of course, higher order statistics also contains cosmological information. It is convenient to work in the multipole space and define the convergence power spectrum as the harmonic transform of the two-point correlation function. This is usually the most analyzed and studied statistical quantity related to the weak lensing, and we will focus on the convergence power spectra in order to properly compare our results to similar analyses in the literature. However, it should be stressed that, as shown in [38], the convergence power spectrum is only indirectly and partially obtainable from the two-point correlation function.
Future surveys will measure redshifts of billions of galaxies, allowing the possibility of a tomographic reconstruction of the matter distribution. We can hence define the convergence power spectra in each redshift bin and the cross-power spectra:

$$
P_{j k}(\ell)=H_{0}^{3} \int_{0}^{\infty} \frac{d z}{E(z)} W_{i}(z) W_{j}(z) P_{N L}\left[P_{L}\left(\frac{H_{0} \ell}{r(z)}, z\right)\right],
$$

where $P_{N L}$ is the nonlinear matter power spectrum at redshift $z$, obtained by correcting the linear one, $P_{L}$. $W(z)$ is a weighting function:

$$
W_{i}(z)=\frac{3}{2} \Omega_{m}(1+z) \int_{z_{i}}^{z_{i+1}} d z^{\prime} \frac{n_{i}\left(z^{\prime}\right) r\left(z, z^{\prime}\right)}{r\left(0, z^{\prime}\right)},
$$

with subscripts $i$ and $j$ indicating the bins in redshifts. Equation (7) clarifies the cosmological information contained in the weak lensing: the function $W(z)$ encodes the information on how the three-dimensional matter distribution is projected on the sky, while the matter power spectrum quantifies the overall matter distribution.

The observed convergence power spectrum is affected mainly by a systematic arising from the intrinsic ellipticity of galaxies $\gamma_{\mathrm{rms}}^{2}$. This uncertainties can be reduced by averaging over a large number of sources. The observed convergence power spectra will hence be

$$
C_{j k}=P_{j k}+\delta_{j k} \gamma_{\mathrm{rms}}^{2} \tilde{n}_{j}^{-1},
$$

where $\tilde{n}_{j}$ is the number of sources per steradian in the $j$ th bin.

\section{CMB LENSING EXTRACTION}

In addition to galaxy weak lensing, we include the information derived from $\mathrm{CMB}$ lensing extraction.

Gravitational CMB lensing, as already shown in Ref. [39], can improve significantly the CMB constraints on several cosmological parameters, since it is strongly connected with the growth of perturbations and gravitational potentials at redshifts $z<1$ and, therefore, it can break important degeneracies. The lensing deflection field $d$ can be related to the lensing potential $\phi$ as $d=\nabla \phi[40]$. In harmonic space, the deflection and lensing potential multipoles follow:

$$
d_{\ell}^{m}=-i \sqrt{\ell(\ell+1)} \phi_{\ell}^{m},
$$

and therefore, the power spectra $C_{\ell}^{d d} \equiv\left\langle d_{\ell}^{m} d_{\ell}^{m *}\right\rangle$ and $C_{\ell}^{\phi \phi} \equiv\left\langle\phi_{\ell}^{m} \phi_{\ell}^{m *}\right\rangle$ are related through

$$
C_{\ell}^{d d}=\ell(\ell+1) C_{\ell}^{\phi \phi} .
$$

Gravitational lensing introduces a correlation between different CMB multipoles (that otherwise would be fully uncorrelated) through the relation 


$$
\left\langle a_{\ell}^{m} b_{\ell^{\prime}}^{m^{\prime}}\right\rangle=(-1)^{m} \delta_{m}^{m^{\prime}} \delta_{\ell}^{\ell^{\prime}} C_{\ell}^{a b}+\sum_{L M} \Xi_{\ell \ell^{\prime} L}^{m m^{\prime} M} \phi_{L}^{M},
$$

where $a$ and $b$ are the $T, E, B$ modes and $\Xi$ is a linear combination of the unlensed power spectra $\tilde{C}_{\ell}^{a b}$ (see [41] for details).

In order to obtain the deflection power spectrum from the observed $C_{\ell}^{a b}$, we have to invert Eq. (12), defining a quadratic estimator for the deflection field given by

$$
d(a, b)_{L}^{M}=n_{L}^{a b} \sum_{\ell \ell^{\prime} m m^{\prime}} W(a, b)_{\ell \ell^{\prime} L}^{m m^{\prime} M} a_{\ell}^{m} b_{\ell^{\prime}}^{m^{\prime}}
$$

where $n_{L}^{a b}$ is a normalization factor needed to construct an unbiased estimator $[d(a, b)$ must satisfy Eq. (10)]. This estimator has a variance

$$
\left\langle d(a, b)_{L}^{M *} d\left(a^{\prime}, b^{\prime}\right)_{L^{\prime}}^{M^{\prime}}\right\rangle \equiv \delta_{L}^{L^{\prime}} \delta_{M}^{M^{\prime}}\left(C_{L}^{d d}+N_{L}^{a a^{\prime} b b^{\prime}}\right)
$$

that depends on the choice of the weighting factor $W$ and leads to a noise $N_{L}^{a a^{\prime} b b^{\prime}}$ on the deflection power spectrum $C_{L}^{d d}$ obtained through this method. The choice of $W$ and the particular lensing estimator we employ will be described in the next section.

\section{FUTURE DATA ANALYSIS}

\section{A. Galaxy weak-lensing data}

Future weak-lensing surveys will measure photometric redshifts of billions of galaxies allowing the possibility of a 3D weak-lensing analysis (e.g. [42-45]) or a tomographic reconstruction of growth of structures as a function of time through a binning of the redshift distribution of galaxies, with a considerable gain of cosmological information (e.g. on neutrinos [46], dark energy [45], the growth of structure [47,48], and the mapping of the dark matter distribution as a function of redshift [49]).

Here we use typical specifications for future weaklensing surveys like the Euclid experiment, observing about 35 galaxies per square arcminute in the redshift range $0<z<2$ with an uncertainty of about $\sigma_{z}=$ $0.03(1+z)$ (see [22]), to build a mock data set of convergence power spectra. Table I shows the number of galaxies per arcminute ${ }^{-2}\left(n_{\text {gal }}\right)$, redshift range, $f_{\text {sky }}$, and intrinsic ellipticity for this survey. The expected $1 \sigma$ uncertainty on the convergence power spectra $P(\ell)$ is given by [50]

TABLE I. Specifications for the Euclid-like survey considered in this paper. The table shows the number of galaxies per square arcminute $\left(n_{\text {gal }}\right)$, redshift range, $f_{\text {sky }}$, and intrinsic ellipticity $\left(\gamma_{\text {rms }}^{2}\right)$.

\begin{tabular}{lccc}
\hline \hline$n_{\text {gal }}\left(\operatorname{arcmin}^{-2}\right)$ & Redshift & $f_{\text {sky }}$ & $\gamma_{\text {rms }}^{2}$ \\
\hline 35 & $0<z<2$ & 0.5 & 0.22 \\
\hline \hline
\end{tabular}

$$
\sigma_{\ell}=\sqrt{\frac{2}{(2 \ell+1) f_{\mathrm{sky}} \Delta_{\ell}}}\left(P(\ell)+\frac{\gamma_{\mathrm{rms}}^{2}}{n_{\mathrm{gal}}}\right),
$$

where $\Delta_{\ell}$ is the bin used to generate data. Here we choose $\Delta_{\ell}=1$ for the range $2<\ell<100$ and $\Delta_{\ell}=40$ for $100<$ $\ell<1500$. For the convergence power spectra we use $\ell_{\max }=1500$ in order to exclude the scales where the nonlinear growth of the structure is more relevant and the shape of the nonlinear matter power spectra is, as a consequence, more uncertain (see [51]). We describe the galaxy distribution of the Euclid survey as in [52], $n(z) \propto$ $z^{2} \exp \left(-\left(z / z_{0}\right)^{1} \times 5\right)$, where $z_{0}$ is set by the median redshift of the sources, $z_{0}=z_{m} / 1.41$. Here we calculate the power spectra assuming a median redshift $z_{m}=1$. Although this assumption is reasonable for Euclid, it is known that the parameters that control the shape of the distribution function may have strong degeneracies with some cosmological parameters such as matter density, $\sigma_{8}$, and the spectral index [53]. However, we conduct an analysis by also varying the value of $z_{m}$, finding no significant variations in the results (see below).

In one case we also show constraints achievable with tomography, dividing the distribution $n(z)$ in three equal redshift bins. The distribution we are using is shown in Fig. 1, normalized so that $\int n(z) d z=1$, together with the distributions of each redshift bin. As said above, Euclid will observe about 35 galaxies per square arcminute, corresponding to a total of $\sim 2.5 \times 10^{9}$ galaxies. In the tomographical analysis, each one of the bins in Fig. 1 contains, respectively, $25 \%, 51 \%$, and $26 \%$ of the sources. As expected, using tomography, we find an improvement on the cosmological parameters with respect to the single redshift analysis. However, in this first-order analysis we are not considering other systematic effects such as intrinsic alignments of galaxies, selection effects, and shear measurement errors due to uncertainties in the point spread

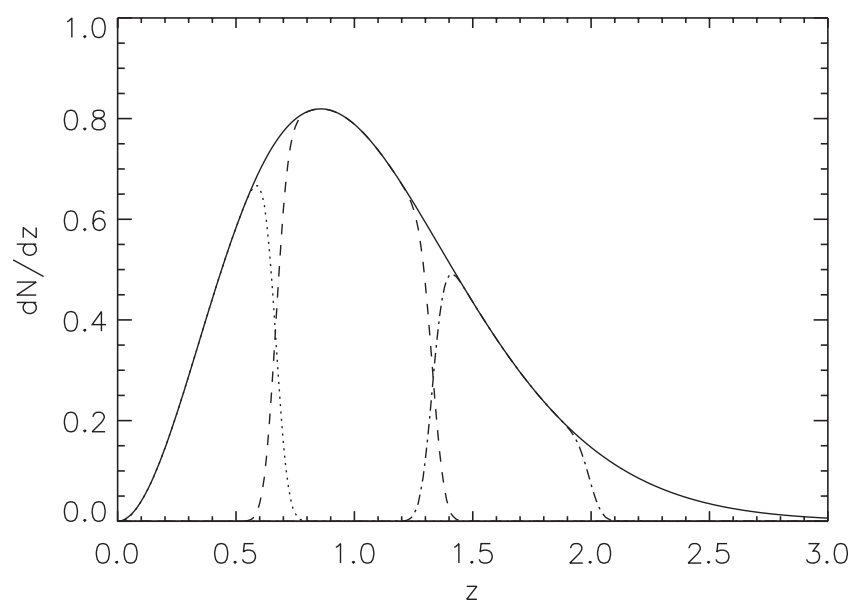

FIG. 1. Redshift distribution of the sources used in this analysis and galaxy distributions in each redshift bin used for the tomography. 
function (PSF) determination. Future real data analysis will require the complete treatment of these effects in order to avoid biases on the cosmological parameters. Moreover, the uncertainty we are assuming on the redshift is the most optimistic value for Euclid, and we note also that the intrinsic ellipticity value of Table I is probably redshift dependent, and may be higher for the fainter galaxies at higher redshifts. For all these reasons we use the convergence power spectra calculated at a single redshift to do most of our forecasts, in order to be more conservative.

\section{B. CMB data}

We create a full mock CMB data set (temperature, $E$-polarization mode, and lensing deflection field) with noise properties consistent with the Planck [54] experiment (see Table II for specifications).

We consider for each channel a detector noise of $w^{-1}=$ $(\theta \sigma)^{2}$, where $\theta$ is the full-width at half-maximum (FWHM) of the beam, assuming a Gaussian profile, and $\sigma$ is the temperature sensitivity $\Delta T$ (see Table II for the polarization sensitivity). We therefore add to each $C_{\ell}$ fiducial spectra a noise spectrum given by

$$
N_{\ell}=w^{-1} \exp \left(\ell(\ell+1) / \ell_{b}^{2}\right)
$$

where $\ell_{b}$ is given by $\ell_{b} \equiv \sqrt{8 \ln 2} / \theta$.

We make use of the method presented in [41] to construct the weighting factor $W$ of Eq. (13). In that paper, the authors chose $W$ to be a function of the power spectra $C_{\ell}^{a b}$, which include both CMB lensing and primary anisotropy contributions. This choice leads to five quadratic estimators, with $a b=T T, T E, E E, E B, T B$; the $B B$ case is excluded because the method of Ref. [41] is only valid when the lensing contribution is negligible compared to the primary anisotropy, an assumption that fails for the $B$ modes in the case of Planck.

The five quadratic estimators can be combined into a minimum variance estimator which provides the noise on the deflection field power spectrum $C_{\ell}^{d d}$ :

$$
N_{\ell}^{d d}=\frac{1}{\sum_{a a^{\prime} b b^{\prime}}\left(N_{\ell}^{a b a^{\prime} b^{\prime}}\right)^{-1}} .
$$

TABLE II. Planck experimental specifications. Channel frequency is given in GHz, FWHM in arcminutes, and the temperature sensitivity per pixel in $\mu K / K$. The polarization sensitivity is $\Delta E / E=\Delta B / B=\sqrt{2} \Delta T / T$.

\begin{tabular}{lccc}
\hline \hline Experiment & Channel & FWHM & $\Delta T / T$ \\
\hline Planck & 70 & $14^{\prime}$ & 4.7 \\
& 100 & $10^{\prime}$ & 2.5 \\
& 143 & $7.1^{\prime}$ & 2.2 \\
$f_{\text {sky }}=0.85$ & & & \\
\hline \hline
\end{tabular}

We compute the minimum variance lensing noise for the Planck experiment by means of a publicly available routine (see Ref. [55]). The data sets (which include the lensing deflection power spectrum) are analyzed with a full-sky exact likelihood routine available and are available in the same reference.

\section{Analysis method}

We perform two different analyses. First, we evaluate the achievable constraints on the $f(R)$ parameter $\lambda_{1}^{2}$ and on the more general scalar-tensor parametrization including also $\beta_{1}$ and $s$. Second, we investigate the effects of a wrong assumption about the gravity framework on the cosmological parameters, by generating an $f(R)$ data set with a nonzero $\lambda_{1}^{2}$ fiducial value but analyzing it assuming $\Lambda \mathrm{CDM}$ and $\lambda_{1}^{2}=0 \mathrm{Mpc}^{2}$.

We perform a Monte Carlo Markov chain analysis based on the publicly available package COSMOMC [56], with a convergence diagnostic using the Gelman and Rubin statistics.

We sample the following set of cosmological parameters, adopting flat priors on them: the baryon and cold dark matter densities $\Omega_{b} h^{2}$ and $\Omega_{c} h^{2}$, the ratio of the sound horizon to the angular diameter distance at decoupling $\theta_{s}$, the scalar spectral index $n_{s}$, the overall normalization of the spectrum $A_{s}$ at $k=0.002 \mathrm{Mpc}^{-1}$, the optical depth to reionization $\tau$, and, finally, the gravity modification parameters $\lambda_{1}^{2}, \beta_{1}$, and $s$.

The fiducial model for the standard cosmological parameters is the best fit from the Wilkinson Microwave Anisotropy Probe seven-year analysis of Ref. [57], with $\Omega_{b} h^{2}=0.02258, \quad \Omega_{c} h^{2}=0.1109, \quad n_{s}=0.963$, $\tau=0.088, A_{s}=2.43 \times 10^{-9}, \Theta=1.0388$.

For modified gravity parameters, we first assume a fiducial value $\lambda_{1}^{2}=0 \mathrm{Mpc}^{2}$ and fix $\beta_{1}=1.33$ and $s=4$

TABLE III. The $68 \%$ C.L. errors on cosmological parameters. Upper limits on $\lambda_{1}^{2}$ are $95 \%$ C.L. constraints. In the third column we show constraints on the cosmological parameters when fitting the data assuming general relativity, i.e. fixing $\lambda_{1}^{2}=0 \mathrm{Mpc}^{2}$.

\begin{tabular}{lccc}
\hline \hline & Planck & \multicolumn{2}{c}{ Planck + Euclid } \\
\hline Fiducial: & $\lambda_{1}^{2}=0$ & $\lambda_{1}^{2}=0$ & $\lambda_{1}^{2}=0$ \\
Model: & Varying $\lambda_{1}^{2}$ & Varying $\lambda_{1}^{2}$ & Fixed $\lambda_{1}^{2}$ \\
Parameter & & & \\
$\Delta\left(\Omega_{b} h^{2}\right)$ & 0.00013 & 0.00011 & 0.00010 \\
$\Delta\left(\Omega_{c} h^{2}\right)$ & 0.0010 & 0.00073 & 0.00057 \\
$\Delta\left(\theta_{s}\right)$ & 0.00027 & 0.00025 & 0.00023 \\
$\Delta(\tau)$ & 0.0041 & 0.0030 & 0.0026 \\
$\Delta\left(n_{s}\right)$ & 0.0031 & 0.0029 & 0.0027 \\
$\Delta\left(\log \left[10^{10} A_{s}\right]\right)$ & 0.013 & 0.0091 & 0.0091 \\
$\Delta\left(H_{0}\right)$ & 0.50 & 0.38 & 0.29 \\
$\Delta\left(\Omega_{\Lambda}\right)$ & 0.0050 & 0.0040 & 0.0031 \\
$\lambda_{1}^{2}\left(\mathrm{Mpc}^{2}\right)$ & $<2.42 \times 10^{4}$ & $<2.9 \times 10^{2}$ & $\cdots$ \\
\hline \hline
\end{tabular}



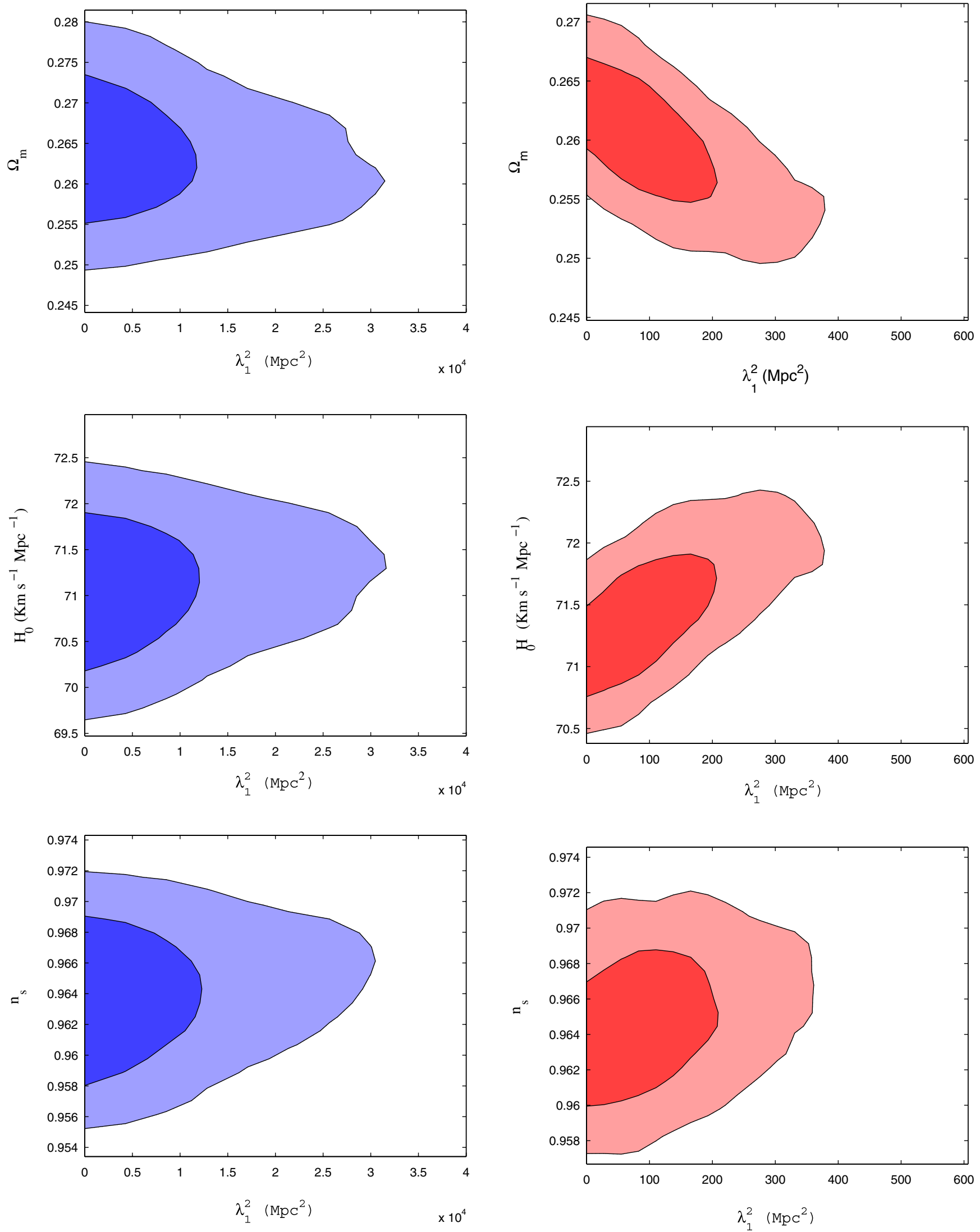

FIG. 2 (color online). Two-dimensional contour plots showing the degeneracies at $68 \%$ and $95 \%$ confidence levels for Planck on the left (blue contours) and Planck + Euclid on the right (red contours). Notice the different scale for the abscissae. 
to test the constraints achievable on the $f(R)$ model. We then repeat the analysis allowing $\beta_{1}$ and $s$ to vary. Furthermore, to investigate the ability of the combination of Planck and Euclid data to detect a hypothetical modified gravity scenario, we study a model with fiducial $\lambda_{1}^{2}=$ $300 \mathrm{Mpc}^{2}$, leaving $\lambda_{1}^{2}, \beta_{1}$, and $s$ as free variable parameters and allowing them to vary in the ranges $0 \leq \lambda_{1}^{2} \leq 10^{6}$, $0.1 \leq \beta_{1} \leq 2$, and $1 \leq s \leq 4$. Finally, we analyze a data set with a fiducial value $\lambda_{1}^{2}=300 \mathrm{Mpc}^{2}$, but wrongly assume a $\Lambda \mathrm{CDM}$ scenario with $\lambda_{1}^{2}=0 \mathrm{Mpc}^{2}$. This will let us investigate the bias introduced on cosmological parameter inference from a wrong assumption about the gravity model.

\section{RESULTS}

In Table III we show the MCMC constraints at 68\% C.L. for the $f(R)$ case for Planck alone and for Planck combined with Euclid. For this last case we also fit the data, fixing $\lambda_{1}^{2}$ to 0 , thus performing a standard analysis in a general relativity framework, in order to show the importance of the degeneracies introduced by $\lambda_{1}^{2}$ on the other cosmological parameter errors. The parameters mostly correlated with modified gravity are $H_{0}$ and $\Omega_{c} h^{2}$ (see also Fig. 2) because these parameters strongly affect the lensing convergence power spectrum as well as $\lambda_{1}^{2}$ through $P(k, z)$. As expected, in fact, when assuming general relativity we find strong improvements on the errors on these parameters for the combination Planck + Euclid in comparison to the varying $\lambda_{1}^{2}$ analysis. We note that the constraints on the standard cosmological parameters are in good agreement with those reported in [21].

In Fig. 2 we show the $68 \%$ and $95 \%$ confidence level 2D likelihood contour plots in the $\Omega_{m}-\lambda_{1}^{2}, H_{0}-\lambda_{1}^{2}$, and $n_{s}-\lambda_{1}^{2}$ planes, for Planck on the left (blue) and Planck + Euclid on the right (red). As one can see, the inclusion of Euclid data can improve constraints on the standard cosmological parameters from $10 \%$ to $30 \%$, with the most important improvements on the dark matter physical density and the Hubble parameter to which the weak lensing is of course very sensitive, as shown in Sec. III. Concerning modifications to gravity, Euclid data are decisive to constrain $\lambda_{1}^{2}$, improving the $95 \%$ C.L. upper limit by 2 orders of magnitude, thanks to the characteristic effect of the modified theory of gravity on the growth of structures.

As stated above, a big advantage of future surveys is the possibility to tomographically reconstruct the matter distribution. Hence, we repeated the analysis considering also a tomographic survey, splitting the galaxy distribution into three redshift bins; this way, as shown in Table IV, we obtain $\mathrm{a} \approx 30 \%$ improvement on constraints, confirming the importance of tomography for future data analysis.

Nevertheless, in this work we are focusing on the relative improvement achievable with Euclid data with respect to Planck data alone, rather than on the absolute uncertainty on the parameters. Indeed, we are not taking into
TABLE IV. The $68 \%$ C.L. errors on cosmological parameters and upper limits (at 95\% C.L.) on $\lambda_{1}^{2}$ with and without a tomographic survey.

\begin{tabular}{lcc}
\hline \hline & \multicolumn{2}{c}{ Planck + Euclid } \\
\hline Parameter & No tomography & With tomography \\
$\Delta\left(\Omega_{b} h^{2}\right)$ & 0.00011 & 0.00010 \\
$\Delta\left(\Omega_{c} h^{2}\right)$ & 0.00073 & 0.00063 \\
$\Delta\left(\theta_{s}\right)$ & 0.00025 & 0.00024 \\
$\Delta(\tau)$ & 0.0030 & 0.0026 \\
$\Delta\left(n_{s}\right)$ & 0.0029 & 0.0021 \\
$\Delta\left(\log \left[10^{10} A_{s}\right]\right)$ & 0.0091 & 0.007 \\
$\Delta\left(H_{0}\right)$ & 0.38 & 0.33 \\
$\Delta\left(\Omega_{\Lambda}\right)$ & 0.0040 & 0.0035 \\
$\lambda_{1}^{2}\left(\mathrm{Mpc}^{2}\right)$ & $<2.9 \times 10^{2}$ & $<2.02 \times 10^{2}$ \\
\hline \hline
\end{tabular}

account several systematic effects (such as PSF or intrinsic alignment) that may weaken constraints. Hence, we choose to use the nontomographic analysis as a conservative estimation of the constraints.

Furthermore, we also performed the analysis with a different median redshift $(\bar{z}=1.5)$ in order to check how possible degeneracies between $\bar{z}$ and other parameters may affect the results. These results are also shown in Table V, and they are very close to the results obtained with $\bar{z}=1$; thus the assumption of $\bar{z}=1$ should not affect the analysis.

Moreover, when analyzing the $f(R)$ mock data sets with $\lambda_{1}^{2}=300 \mathrm{Mpc}^{2}$ as the fiducial model, assuming $\lambda_{1}^{2}=0 \mathrm{Mpc}^{2}$ we found a consistent bias in the recovered best-fit value of the cosmological parameters due to the degeneracies between $\lambda_{1}^{2}$ and the other parameters. As it can be seen from the comparison of Figs. 2 and 3 and from Table VI, the shift in the best-fit values is, as expected, along the degeneracy direction of the parameters with $\lambda_{1}^{2}$, for example, for $n_{s}, H_{0}$, and $\Omega_{m}$. These results show that for even small modifications to gravity, the best-fit values recovered by wrongly assuming general relativity are more than $68 \%$ C.L. (for some parameters, more than 95\% C.L.) away from the correct fiducial values, and may cause an underestimation of $n_{s}$ and $H_{0}(\sim 1-\sigma$ away from the

TABLE V. The $68 \%$ C.L. errors on cosmological parameters and upper limits (at 95\% C.L.) on $\lambda_{1}^{2}$ using $\bar{z}=1$ and $\bar{z}=1.5$.

\begin{tabular}{lcc}
\hline \hline & \multicolumn{2}{c}{ Planck + Euclid } \\
\hline Parameter & $\bar{z}=1$ & $\bar{z}=1.5$ \\
$\Delta\left(\Omega_{b} h^{2}\right)$ & 0.00011 & 0.00012 \\
$\Delta\left(\Omega_{c} h^{2}\right)$ & 0.00073 & 0.00076 \\
$\Delta\left(\theta_{s}\right)$ & 0.00025 & 0.00025 \\
$\Delta(\tau)$ & 0.0030 & 0.0032 \\
$\Delta\left(n_{s}\right)$ & 0.0029 & 0.0029 \\
$\Delta\left(\log \left[10^{10} A_{s}\right]\right)$ & 0.0091 & 0.009 \\
$\Delta\left(H_{0}\right)$ & 0.38 & 0.40 \\
$\Delta\left(\Omega_{\Lambda}\right)$ & 0.0040 & 0.0042 \\
$\lambda_{1}^{2}\left(\mathrm{Mpc}^{2}\right)$ & $<2.9 \times 10^{2}$ & $<2.67 \times 10^{2}$ \\
\hline \hline
\end{tabular}



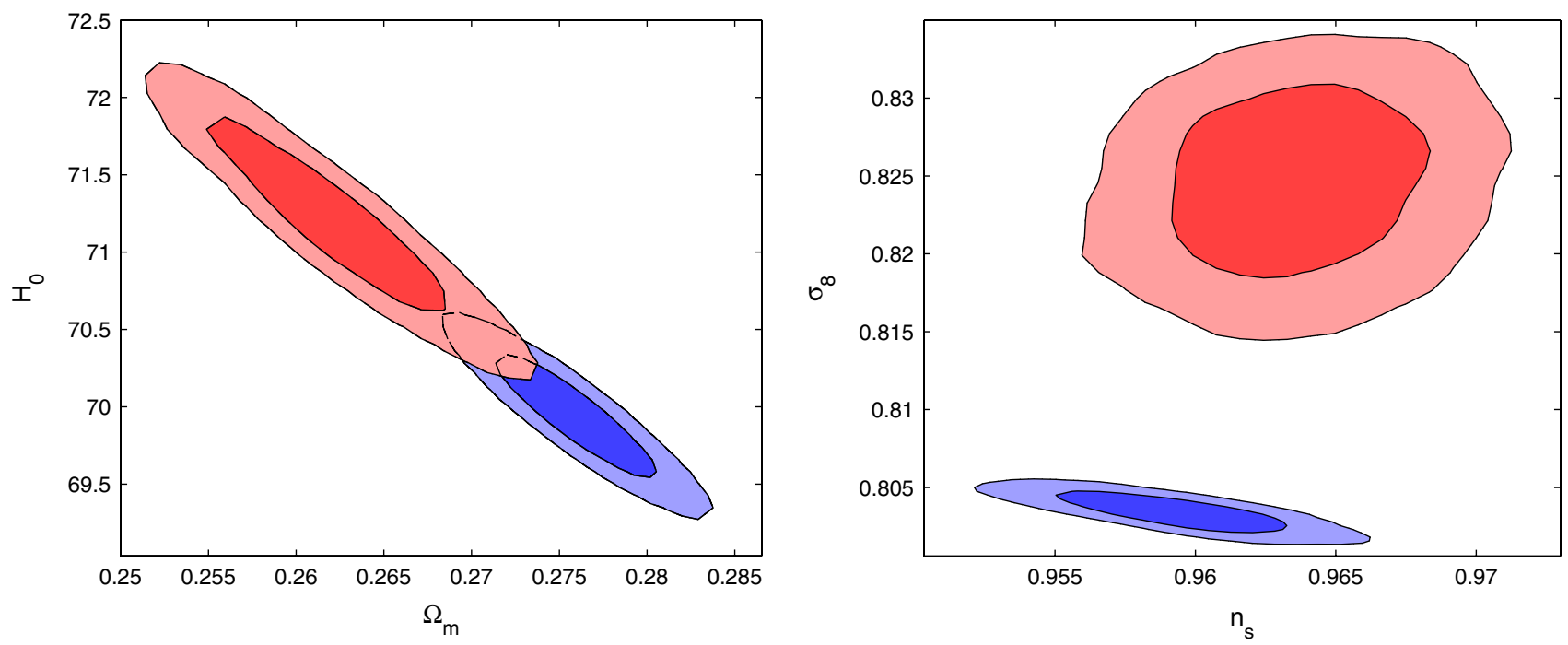

FIG. 3 (color online). Two-dimensional contour plots showing the degeneracies at $68 \%$ and $95 \%$ confidence levels for Planck + Euclid assuming an $f(R)$ fiducial cosmology with $\lambda_{1}^{2}=300 \mathrm{Mpc}^{2}$, considering an analysis with $\lambda_{1}^{2}$ fixed to 0 (blue contours) or allowing it to vary (red contours).

fiducial value), and of $\sigma_{8}(\sim 5-\sigma)$. More generally, as shown in Table VI, all parameters are affected.

We conclude, hence, that future analyses of high precision data from Euclid and Planck need to consider possible deviations from general relativity in order not to bias the constraints on the cosmological parameters.

We also perform an analysis allowing $\beta_{1}$ and $s$ to vary; in this way we can constrain not only $f(R)$ theories but also more general scalar-tensor models, adding to the standard parameter set the time variation of the new gravitational interaction $s$ and the coupling with matter $\beta_{1}$.

We perform this analysis assuming as a fiducial model a $f(R)$ theory with $\lambda_{1}^{2}=3.0 \times 10^{4} \mathrm{Mpc}^{2}$ and $\beta_{1}=4 / 3$.

In Table VII we report the $68 \%$ C.L. errors on the standard cosmological parameters, plus the coupling parameter $\beta_{1}$. Performing a linear analysis, with a fiducial value of $\lambda_{1}^{2}=3 \times 10^{4}$, we obtain constraints on $\beta_{1}$ with $\Delta\left(\beta_{1}\right)=0.038$ at $68 \%$ C.L., therefore potentially discriminating between modified theories of gravity and excluding the $\beta_{1}=1$ case (corresponding to the standard $\Lambda \mathrm{CDM}$ model) at more than $5-\sigma$ from a combination of Planck + Euclid data (only $2-\sigma$ for Planck alone).

The strong correlation present between $\beta_{1}$ and $\lambda_{1}^{2}$ [see Eq. (4)] implies that, choosing a lower $\lambda_{1}^{2}$ fiducial value for an $f(R)$ model, the same variation of $\beta_{1}$ leads to smaller modifications of CMB power spectra, and therefore we can expect weaker bounds on the coupling parameter. In order to verify this behavior we made three analyses, fixing $s=4$ and choosing three different fiducial values for $\lambda_{1}^{2}$ : $3 \times 10^{2}, 3 \times 10^{3}$, and $3 \times 10^{4} \mathrm{Mpc}^{2}$. The respectively obtained $\beta_{1} 68 \%$ C.L. errors are $0.11,0.052$, and 0.035 , confirming the decreasing expected accuracy on $\beta_{1}$ for smaller fiducial values of $\lambda_{1}^{2}$.

The future constraints presented in this paper are obtained using a MCMC approach. Since most of the forecasts present in the literature on $f(R)$ theories are obtained

TABLE VI. The best-fit value and $68 \%$ C.L. errors on cosmological parameters for the case with a fiducial model $\lambda_{1}^{2}=300$ fitted with a $\Lambda \mathrm{CDM}$ model, where $\lambda_{1}^{2}=0$ is assumed.

\begin{tabular}{lccc}
\hline \hline & Planck + Euclid & Planck + Euclid & Fiducial values \\
\hline Model: & $\lambda_{1}^{2}=0$ & Varying $\lambda_{1}^{2}$ & \\
Parameter & & & \\
$\Omega_{b} h^{2}$ & $0.022326 \pm 0.000096$ & $0.02259 \pm 0.00012$ & 0.02258 \\
$\Omega_{c} h^{2}$ & $0.1126 \pm 0.00055$ & $0.11030 \pm 0.00083$ & 0.1109 \\
$\theta_{s}$ & $1.0392 \pm 0.00023$ & $1.0395 \pm 0.00025$ & 1.0396 \\
$\tau$ & $0.0775 \pm 0.0024$ & $0.08731 \pm 0.0029$ & 0.088 \\
$n_{s}$ & $0.9592 \pm 0.0027$ & $0.9636 \pm 0.0029$ & 0.963 \\
$H_{0}$ & $69.94 \pm 0.27$ & $71.20 \pm 0.42$ & 71.0 \\
$\Omega_{\Lambda}$ & $0.724 \pm 0.003$ & $0.738 \pm 0.005$ & 0.735 \\
$\sigma_{8}$ & $0.8034 \pm 0.0008$ & $0.8245 \pm 0.0039$ & 0.8239 \\
\hline \hline
\end{tabular}


TABLE VII. The $68 \%$ C.L. errors on cosmological parameters and $\beta_{1}$. We do not show limits on $\lambda_{1}^{2}$ and $s$ because this kind of analysis does not allow us to constrain them (see text).

\begin{tabular}{lcc}
\hline \hline & Planck & Planck + Euclid \\
\hline Fiducial: & $\lambda_{1}^{2}=3.0 \times 10^{4}$ & $\lambda_{1}^{2}=3.0 \times 10^{4}$ \\
Parameter & 0.00013 & \\
$\Delta\left(\Omega_{b} h^{2}\right)$ & 0.0011 & 0.00011 \\
$\Delta\left(\Omega_{c} h^{2}\right)$ & 0.00026 & 0.00082 \\
$\Delta\left(\theta_{s}\right)$ & 0.0043 & 0.00025 \\
$\Delta(\tau)$ & 0.0033 & 0.0040 \\
$\Delta\left(n_{s}\right)$ & 0.014 & 0.0029 \\
$\Delta\left(\log \left[10^{10} A_{s}\right]\right)$ & 0.54 & 0.011 \\
$\Delta\left(H_{0}\right)$ & 0.0060 & 0.40 \\
$\Delta\left(\Omega_{\Lambda}\right)$ & 0.13 & 0.0045 \\
$\Delta\left(\beta_{1}\right)$ & Unconstrained & 0.038 \\
$\lambda_{1}^{2}$ & Unconstrained & Unconstrained \\
$s$ & & Unconstrained \\
\hline \hline
\end{tabular}

using a Fisher matrix analysis, it is useful to compare our results with those predicted by a Fisher matrix approach. We therefore perform a Fisher matrix analysis for Planck and Planck + Euclid (see $[46,58,59]$ ) assuming a $\Lambda$ CDM fiducial model, and we compare the results with those in Table III.

We find that for Planck alone the error on $\lambda_{1}$ is underestimated by a factor $\sim 3$, while the error is closer to the MCMC result for the Planck + Euclid case (underestimated by a factor $\sim 1.2$ ).

\section{CONCLUSIONS}

In this paper we forecasted the ability of future weaklensing surveys such as Euclid to constrain modifications to gravity. We restricted our analysis to models that could mimic a cosmological constant in the expansion of the Universe and can therefore be discriminated by only looking at the growth of perturbations. We have found that Euclid could improve the constraints on these models by nearly 2 orders of magnitude with respect to the constraints achievable by the Planck CMB satellite alone. We have also discussed the degeneracies among the parameters, and we found that neglecting the possibility of gravity modifications can strongly affect the constraints from Euclid on parameters such as the Hubble constant $H_{0}, \Omega_{m}$, and the amplitude of rms fluctuations $\sigma_{8}$. In this paper we found that considering more general expansion histories would further relax our constraints and increase the degeneracies between the parameters. However, other observables can be considered, such as baryonic acoustic oscillation and luminosity distances of high redshift supernovae, to further probe the value of $w$ and its redshift dependence.

\section{ACKNOWLEDGMENTS}

It is a pleasure to thank Adam Amara and Luca Amendola for useful comments and suggestions. We also thank Gong-Bo Zhao for the latest version of the MGCAMB code. Support was given by the Italian Space Agency through the ASI Contract No. Euclid-IC (I/031/10/0).
[1] S. Tsujikawa, arXiv:1004.1493.

[2] A. Silvestri and M. Trodden, Rep. Prog. Phys. 72, 096901 (2009).

[3] E. W. Kolb, V. Marra, and S. Matarrese, Gen. Relativ. Gravit. 42, 1399 (2009).

[4] V. Marra, E. W. Kolb, S. Matarrese, and A. Riotto, Phys. Rev. D 76, 123004 (2007).

[5] P. Serra, A. Cooray, D. E. Holz, A. Melchiorri, S. Pandolfi, and D. Sarkar, Phys. Rev. D 80, 121302 (2009).

[6] T. Padmanabhan, Phys. Rep. 380, 235 (2003).

[7] P. J.E. Peebles and B. Ratra, Rev. Mod. Phys. 75, 559 (2003).

[8] I. Laszlo and R. Bean, Phys. Rev. D 77, 024048 (2008).

[9] L. Amendola, M. Kunz, and D. Sapone, J. Cosmol. Astropart. Phys. 04 (2008) 013.

[10] E. V. Linder and R. N. Cahn, Astropart. Phys. 28, 481 (2007).

[11] E. J. Copeland, M. Sami, and S. Tsujikawa, Int. J. Mod. Phys. D 15, 1753 (2006).

[12] M. Bartelmann and P. Schneider, Phys. Rep. 340, 291 (2001).
[13] A. Refregier, Annu. Rev. Astron. Astrophys. 41, 645 (2003).

[14] D. Munshi, P. Valageas, L. Van Waerbeke, and A. Heavens, Phys. Rep. 462, 67 (2008).

[15] S. F. Daniel, E. V. Linder, T. L. Smith, R. R. Caldwell, A. Cooray, A. Leauthaud, and L. Lombriser, Phys. Rev. D 81, 123508 (2010).

[16] L. Lombriser, A. Slosar, U. Seljak, and W. Hu, arXiv:1003.3009.

[17] R. Bean and M. Tangmatitham, Phys. Rev. D 81, 083534 (2010).

[18] G. B. Zhao et al., Phys. Rev. D 81, 103510 (2010).

[19] S. F. Daniel, R. R. Caldwell, A. Cooray, and A. Melchiorri, Phys. Rev. D 77, 103513 (2008).

[20] S. F. Daniel et al., Phys. Rev. D 80, 023532 (2009).

[21] A. Refregier, A. Amara, T.D. Kitching, A. Rassat, R. Scaramella, and J. Weller (f.t.E. Consortium), arXiv:1001.0061.

[22] A. Refregier et al., Proc. SPIE Int. Soc. Opt. Eng. 6265, 62651Y (2006); .

[23] N. Gehrels, arXiv:1008.4936. 
[24] S. A. Thomas, F. B. Abdalla, and J. Weller, Mon. Not. R. Astron. Soc. 395, 197 (2009).

[25] J. Q. Xia, Phys. Rev. D 79, 103527 (2009).

[26] G. B. Zhao, L. Pogosian, A. Silvestri, and J. Zylberberg, Phys. Rev. D 79, 083513 (2009).

[27] A. F. Heavens, T. D. Kitching, and L. Verde, Mon. Not. R. Astron. Soc. 380, 1029 (2007).

[28] E. Calabrese, A. Cooray, M. Martinelli, A. Melchiorri, L. Pagano, A. Slosar, and G. F. Smoot, Phys. Rev. D 80, 103516 (2009).

[29] P. Serra, A. Cooray, S. F. Daniel, R. Caldwell, and A. Melchiorri, Phys. Rev. D 79, 101301 (2009).

[30] W. Hu and I. Sawicki, Phys. Rev. D 76, 064004 (2007).

[31] http://www.sfu.ca/ gza5/MGCAMB.html.

[32] E. Bertschinger and P. Zukin, Phys. Rev. D 78, 024015 (2008).

[33] J. Khoury and A. Weltman, Phys. Rev. Lett. 93, 171104 (2004).

[34] D. Wands, Classical Quantum Gravity 11, 269 (1994).

[35] G. Magnano and L. M. Sokolowski, Phys. Rev. D 50, 5039 (1994).

[36] T. Giannantonio, M. Martinelli, A. Silvestri, and A. Melchiorri, J. Cosmol. Astropart. Phys. 04 (2010) 030.

[37] D. Huterer, Gen. Relativ. Gravit. 42, 2177 (2010).

[38] P. Schneider, L. van Waerbeke, M. Kilbinger, and Y. Mellier, Astron. Astrophys. 396, 1 (2002).

[39] L. Perotto, J. Lesgourgues, S. Hannestad, H. Tu, and Y. Y. Y. Wong, J. Cosmol. Astropart. Phys. 10 (2006) 013.

[40] C. M. Hirata and U. Seljak, Phys. Rev. D 68, 083002 (2003).

[41] T. Okamoto and W. Hu, Phys. Rev. D 67, 083002 (2003).
[42] A. F. Heavens, Mon. Not. R. Astron. Soc. 343, 1327 (2003).

[43] P. G. Castro, A. F. Heavens, and T. D. Kitching, Phys. Rev. D 72, 3516 (2005).

[44] A. F. Heavens, T. D. Kitching, and A. N. Taylor, Mon. Not. R. Astron. Soc. 373, 105 (2006).

[45] T. D. Kitching, A. F. Heavens, A. N. Taylor, M. L. Brown, K. Meisenheimer, C. Wolf, M.E. Gray, and D. J. Bacon, Mon. Not. R. Astron. Soc. 376, 771 (2007).

[46] S. Hannestad, H. Tu, and Y. Y. Y. Wong, J. Cosmol. Astropart. Phys. 06 (2006) 025.

[47] D. Bacon et al., Mon. Not. R. Astron. Soc. 363, 723 (2005).

[48] R. Massey et al., Astrophys. J. Suppl. Ser. 172, 239 (2007).

[49] A. N. Taylor et al., Mon. Not. R. Astron. Soc. 353, 1176 (2004).

[50] A. R. Cooray, Astron. Astrophys. 348, 31 (1999).

[51] R. E. Smith et al. (Virgo Consortium Collaboration), Mon. Not. R. Astron. Soc. 341, 1311 (2003).

[52] F. B. Abdalla, A. Amara, P. Capak, E. S. Cypriano, O. Lahav, and J. Rhodes, Mon. Not. R. Astron. Soc. 387, 969 (2008).

[53] L. Fu et al., Astron. Astrophys. 479, 9 (2008).

[54] Planck Collaboration, arXiv:astro-ph/0604069.

[55] http://lesgourg.web.cern.ch/lesgourg/codes.html

[56] A. Lewis and S. Bridle, Phys. Rev. D 66, 103511 (2002).

[57] E. Komatsu et al., arXiv:1001.4538.

[58] F. De Bernardis, L. Pagano, P. Serra, A. Melchiorri, and A. Cooray, J. Cosmol. Astropart. Phys. 06 (2008) 013.

[59] J. R. Bond, G. Efstathiou, and M. Tegmark, Mon. Not. R. Astron. Soc. 291, L33 (1997). 